\title{
Risk Factor Assessment of Multidrug-Resistant Tuberculosis
}

\author{
Pant $R,{ }^{1}$ Pandey $K R,{ }^{2}$ Joshi $M,{ }^{1}$ Sharma $S,{ }^{3}$ Pandey $T,{ }^{4}$ Pandey $S^{5}$ \\ ${ }^{1}$ Bhim Hospital, Bhairahawa, ${ }^{2}$ Doti District Hospital, Doti, ${ }^{3}$ Bhim Hospital, Bhairawaha, ${ }^{4}$ Department of Medicine, \\ Tribhuban University Teaching Hospital, Kathmandu, ${ }^{5} \mathrm{Nepal}$ Medical College Teaching Hospital.
}

\section{ABSTRACT}

Background: Despite increasing numbers of MDR TB cases seen in Nepal, a lot remains to be understood about the disease in the local context. We evaluated possible risk factors for MDR TB among patients enrolled for treatment at a district hospital in western Nepal.

Methods: A descriptive case-series study using structured interviews and abstraction of treatment records of all patients registered at the DOTS Plus clinic at Bhim Hospital, Bhairahawa from April 2008 to Dec 2008 was done. Descriptive analysis was done to find out frequencies and relations.

Results: Of the total 31 patients, 22 were males (age range 18-68, median 36.7) and the remaining 9 females (age range 23-56, median 33.7). 27(87\%) of the patients had monthly income below Rs 3000 and 24 (77\%) of them were illiterate. 21(67\%) had missed at least a few weeks of drugs during their previous treatment and $4(13 \%)$ had been marked as defaulters. $6(20 \%)$ had treatment failure. $74 \%$ of the patients were smokers, 2 were HIV positive. $20(58 \%)$ had lived in India for at least 6 months where they had incomplete treatment of TB. 30 (97\%) patients had disclosed their MDR status to their families; however $70 \%$ said they did not do so immediately.

Conclusions: Previous TB treatment, male sex, poverty, migration to India, illiteracy and smoking have been observed in a majority of the cases in this study. These findings need to be corroborated with multi-centre casecontrol studies to bring out nationally relevant risk factors for MDR TB.

Key words: directly observed treatment short course-plus, multi- drug resistance, risk factors, tuberculosis.

\section{INTRODUCTION}

Though the Directly Observed Treatment Short Course (DOTS) program has been operational in Nepal for more than a decade now, the burden of incident cases of TB is yet to register a significant fall. ${ }^{1}$ Moreover, the emergence of Multi Drug Resistant (MDR-defined as TB bacterial resistance to Isoniazid and Rifampicin) tuberculosis bacilli threatens to undo any achievement thus far made in the areas of TB control. ${ }^{2}$ Globally, in 2006 alone, almost 0.5 million cases of MDR-TB are said to have occurred. ${ }^{3}$
Under the current program management guidelines of the NTP, patients treated under DOTS-Plus are: 1) any smear positive CAT-2 failure, 2) CAT-1 failure and culture and Drug Sensitivity Testing (DST) confirmed MDR-TB, 3) any MDR-TB patient household contact who is smear positive with culture and DST confirmed MDR-TB, 4) any MDR-TB patient who is smear positive and culture and DST confirmed MDR-TB. ${ }^{2}$

Adequate study regarding the role of host related factors responsible for the occurrence of MDR-TB has hitherto been lacking in the local context. We conducted this 
study in an attempt to identify possible risk factors for MDR-TB.

\section{METHODS}

This is a descriptive, case-series study done on MDR-TB patients registered for DOTS-Plus treatment at Bhim Hospital- the Rupandehi district hospital at Bhairahawa from December 2008 to January 2009.

Ethical approval was obtained from the hospital ethics committee before the study was done. Interview was conducted in a confidential area at the clinic. Written informed consent was obtained from the participants before the interview. Participants were informed that participation in the study was voluntary and that they could end the interview at any point in time if they chose to do so. Participants were also informed that decision regarding participation in the survey would not have any bearing on their current or future access to services

Inclusion criterion for the study was restricted to all MDR-TB patients registered at the DOTS-Plus clinic at the hospital from April 2008 to Dec 2008. Patients unwilling to participate in the study, or not turning up at the hospital during the study period were excluded from the study.

Multiple variables under demographic factors, socioeconomic factors, TB contact and treatment, life-style factors, migration, co-morbid factors, and disclosure were studied. Data collection was done via structured interviews with the patients during visits to the DOTSPlus clinic; additional information was obtained via chart abstraction at the DOTS-Plus clinic.

Data entry was done using Microsoft Office Excel 2007 and data analysis was done using Statistical Package for Social Sciences (SPSS) version 17.0.

\section{RESULTS}

Although 36 patients had been registered for DOTS-Plus during the period all of them could not be recruited for the study as 3 of them did not turn up at the hospital during the study period and 2 of them refused to participate in the study.

Analysis of all variables for the 31 patients in the study yielded the following results.

Out of these 31 MDR TB patients, 22 (70\%) were male and 9 (30\%) female. Age range was $18-68$ for males and 23 56 for females and mean age for males and females was 36.7 and 33.7 respectively. Mean DOTS-Plus treatment duration was 9.3 months (Figure 1,2).
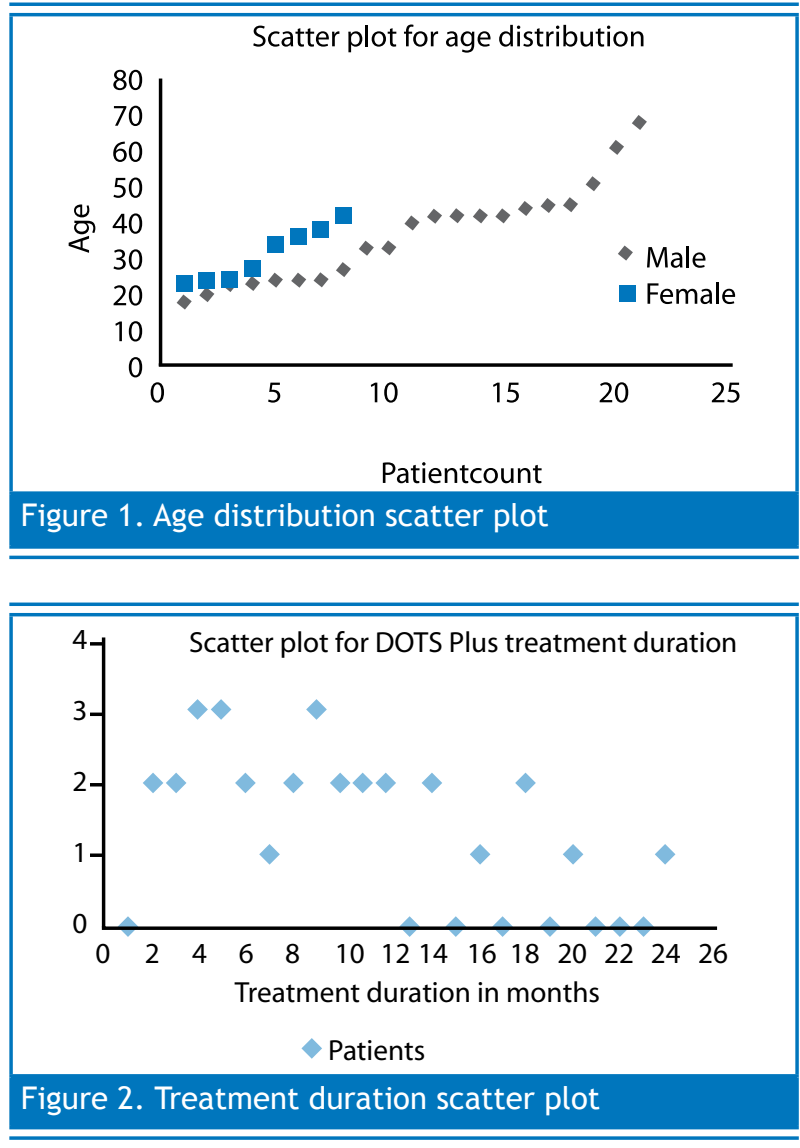

A majority of the participants said their monthly household income was less than NRs 3000 and 27 (87\%) stated that they have been living in a thatched house where 3 or more family members share a single room. Total 24 (77\%) participants were illiterate (Table 1).

Table 1. Monthly income levels of patients

\begin{tabular}{|lcc|}
\hline Income & $\mathrm{n}$ & $\%$ \\
\hline$<$ Rs 3000 & 27 & 87 \\
Rs $3000-$ Rs 6000 & 2 & 6.5 \\
Rs $6000-$ Rs 9000 & 1 & 3.25 \\
$>$ Rs 9000 & 1 & 3.25 \\
Total & 31 & 100 \\
\hline \hline
\end{tabular}

More than half $(54 \%)$ people had contact with a known case of TB (either in their family or friends who had been diagnosed with TB) and among the rest $14(45 \%)$ stated that they had had contact with people who have been coughing for more than 2 weeks but had not had a diagnosis of TB at that point in time.

Out of the total, $4(13 \%)$ had been marked as defaulters and as many as $4 / 5^{\text {th }}$ of the patients had had an incomplete treatment of TB. The remaining $1 / 5^{\text {th }}$ had treatment failure in spite of not missing a single dose of drug during the previous treatment (Table 2). 


\begin{tabular}{|lccc|}
\hline \multicolumn{4}{l}{ Table 2. Previous tuberculosis treatment status } \\
\hline $\begin{array}{l}\text { Previous treatment Status } \\
\text { Missed at least a few weeks of } \\
\text { treatment (A) }\end{array}$ & 21 & 67 \\
$\begin{array}{l}\text { Defaulted on treatment (B) } \\
\text { Had Incomplete TB treatment } \\
\text { of A and B) }\end{array}$ & 4 & 13 \\
$\begin{array}{l}\text { Had MDR TB despite complete } \\
\text { treatment (C) }\end{array}$ & 25 & 80 \\
\hline Total (sum of A B and C) & 6 & 20 \\
\hline \hline
\end{tabular}

All of the patients were taking DOTS-Plus treatment following DOTS CAT-II treatment failure and DST confirmed MDR TB. None of the patients had primary infection with MDR-TB.

As many as 23 (74\%) of the patients had a history of smoking (past as well as present). Among the 23 smokers, 21 said they shared cigarette stick with others. 17 (54\%) MDR patients stated that they were former alcohol drinkers.

Only 2 out of the 31 were infected with HIV. The remaining 29 tested negative for HIV. None of the participants gave a history of prolonged steroid/immunosuppressive therapy intake. None of them gave any history of Diabetes or Hypertension.

More than half (58\%) of the patients had been to India and had lived there for more than 6 months. All 20 of them had been diagnosed of TB in India and started treatment there itself. However none of them could complete their treatment in India due to the cost of treatment and had to discontinue their treatment in India.

Almost all (97\%) of the patients said that they had informed their family that they have MDR-TB and that they need to be in treatment for long duration. Only $1(3 \%)$ had not shared their status with other family members for fear of being socially outcast. However among this $97 \%$, as many as $70 \%$ said they did not inform their family immediately after diagnosis with TB.

\section{DISCUSSION}

Despite the limitations case-series study offers on ascribing risk factors to a disease, notable patterns have emerged through this study. Our study shows, previous TB treatment, male sex, poverty, migration to India, illiteracy, smoking, have demonstrated a significant association with MDR-TB.

All patients in our study had had previous treatment with DOTS. Numerous studies have shown a very significant co-relation between previous treatment of TB and MDRTB. ${ }^{4}$
Elsewhere, prevalence of MDR-TB has been estimated to be up to 10 times higher after unsuccessful treatment of TB. ${ }^{4}$ According to the National Tuberculosis Program's Nepal National Survey 2007, MDR among new cases of TB is $2.9 \%$ and MDR among previously treated cases of TB is at $11.7 \%$ thereby giving a ratio of approximately 4:1 for prevalence of MDR in these two groups. And with as many as $25(80 \%)$ of the cases of MDR following an incomplete previous treatment, this pattern holds true in our study too.

With $70 \%$ of the patients being males, male sex co-relates well with MDR-TB in our study. A meta-analysis of studies done in Europe showed a significant link between male sex and MDR-TB in Western Europe where majority of the MDR patients followed a failed previous treatment. In Eastern Europe where transmission of MDR itself was responsible for MDR TB no sex predilection was observed. ${ }^{4}$ The association between failed treatment and male sex requires further analysis- whether males are less likely to be adherent to treatment than females. Likewise most of the cases in our study are less than 65 years; elsewhere too significant co-relation has been shown between age less than 65 years and the development of MDR TB. The association however has been seen to thin out below the age of 45 years.

At $90 \%$, an overwhelmingly large number of the patients in our study are from lower socio-economic class. Furthermore $3 / 4$ th of the patients are illiterate. Larger case control studies will be required to determine whether or not low socioeconomic status or illiteracy lead to poor adherence to treatment thereby leading to the development of MDR. The European meta-analysis found no linkage between low socioeconomic class or literacy and MDR. In a study in Turkey however, patient's education and MDR TB were found to be inversely related. ${ }^{5}$

Poor housing, over-crowding and homelessness are clear risk factors for TB- environmental attributes that are prevalent among in the terai region. This has led to a greater burden of TB in the terai. ${ }^{6}$ In Rupandehi alone more than 770 patients take DOTS treatment. ${ }^{7}$ Since most TB patients in our case tend to live in shabby, overcrowded housing, it is a natural corollary that the ones with MDR also bear these attributes as the entire cohort of MDR patients in our case belongs to previously treated TB cases.

At $74 \%$, majority of the patients in our study are smokers. The linkage between poor lung health and smoking has been established beyond doubt. Whether or not smoking increases the chances of drug resistance in tuberculosis bacilli is yet to be established. Though more than $50 \%$ of the patients in our cases are alcohol drinkers, larger 
meta-analyses have failed to find linkage between alcohol use and the development of MDR. ${ }^{5}$

Only 2 out of the 31 patients in our study tested positive for HIV. The other 29 had a negative test. The number fits with findings elsewhere- a Peruvian study also found only $1.5 \%$ of MDR TB patients to be infected with HIV. ${ }^{8}$ According to current hypotheses HIV increases the chances of transmission of MDR TB rather than leading to an inadequate treatment. ${ }^{9}$ However in some East European countries, MDR rates are double the normal in HIV positives. ${ }^{3}$ Therefore the HIV positive status and MDR may be events independent of each other in our studyas all the cases developed MDR following inadequate treatment.

Total $20(58 \%)$ of the patients had been to India for a prolonged duration of time and all of them had been diagnosed of TB in India itself; however none of them could complete their treatment in India leading to the development of MDR TB. The relationship between inadequate treatment and MDR TB are so widely recognized that MDR-TB is classified as being due to previous treatment if the patient has been treated before, and attributed to an infection with an MDR strain of $M$. tuberculosis if detected in newly diagnosed patients ${ }^{4}$ Therefore we can safely attribute these cases to inadequate treatment in India. Future programs ought to improve the linkage between TB treatment programs in the two countries so that patients do not end up with inadequate treatment. Furthermore these findings also show that the success of our TB program is also related to the success of the TB program in India.

Given the kind of living conditions the patients have, they can easily be a source of infection to their family members. Therefore disclosure and positive prevention have an important role in the preventing further transmission. Furthermore family support is crucial if these patients are to satisfactorily complete their treatment, thereby preventing the development of XDR TB. At $97 \%$ disclosure of the TB status is satisfactory; however there was a considerable lag between the diagnosis and disclosure. There is enough scope for better counseling and surveillance so that these patients do not transmit their infection to others.

Since DST is not routinely done in new cases of TB it is difficult to say for sure if these cases of MDR TB have been transmitting their infection to others. If they are, the number of MDR TB cases is bound to swell in the future, when these new cases will fail treatment with DOTS. Therefore importance of positive prevention counseling and monitoring among MDR cases can't be exaggerated.

\section{CONCLUSIONS}

A cursory analysis of risk factors for MDR TB has pointed out important associations with risk factors in the local context. The strength and truthfulness of these associations ought to be examined with a multi-centre case-control study, so that nationally relevant risk factors for MDR TB can be unearthed. Such study can be built and extrapolated upon the findings of this caseseries study- by closely examining the associations that have been pointed out by this study. The findings of such a study would add tremendous impetus to the DOTS- Plus program in the country.

\section{REFERENCES}

1. Ministry of Health, Nepal. Report of the National tuberculosis control program. Ministry of Health; 2008

2. National tuberculosis control program. Treatment of multidrug resistant tuberculosis DOTS Plus pilot project manual, Nepal. National tuberculosis control program; 2008.

3. World Health Organisation. Anti tuberculosis drug resistance in the world fourth global report: The WHO/IUATLD global project on anti tuberculosis drug resistance surveillance. World Health Organisation; 2008.

4. Faustini A, Hall AJ, Perucci CA. Risk factors for multi-drug resistant tuberculosis in Europe: a systematic review. Thorax. 2005;61:158-63.

5. Tanrikulu AC, Hosoglu S, Ozekinci T, Abakay A, Gurkan F. Risk factors for drug resistant tuberculosis in southeast Turkey. Trop Doct. 2008;38:91-3.

6. Department of Health Sciences, Government of Nepal. Annual report FY 064/065. Department of Health Sciences; 2008.

7. District Public Health Office. TB control program summary report FY064/065. Rupandehi: District Public Health Office; 2065.

8. Mitnick C, Bayona J, Palacios E, Shin S, Furin J, Alcantara F, et al. Community-Based Therapy for Multidrug-Resistant Tuberculosis in Lima, Peru. N Engl J Med. 2003; 348:119-28.

9. McCrayE, Onorato IM.Theinteraction of humanimmunodeficiency virus and multidrug resistant Mycobacterium tuberculosis. In Bastian I, Portaels F. Multidrug-resistant tuberculosis. Netherlands: Kluwer Academic Publishers; 2000. p. 45-57. 